\title{
Efficient TALEN-mediated myostatin gene editing in goats
}

\author{
Baoli Yu, Rui Lu, Yuguo Yuan, Ting Zhang, Shaozheng Song, Zhengqiang Qi, Bin Shao, Mengmin Zhu, Fei Mi \\ and Yong Cheng
}

\begin{abstract}
Background: Myostatin (MSTN) encodes a negative regulator of skeletal muscle mass that might have applications for promoting muscle growth in livestock. In this study, we aimed to test whether targeted MSTN editing, mediated by transcription activator-like effector nucleases (TALENs), is a viable approach to create myostatin-modified goats (Capra hircus).

Results: We obtained a pair of TALENs (MTAL-2) that could recognize and cut the targeted MSTN site in the goat genome. Fibroblasts from pedigreed goats were co-transfected with MTAL-2, and 272 monoclonal cell strains were confirmed to have mono- or bi-allelic mutations in MSTN. Ten cell strains with different genotypes were used as donor cells for somatic cell nuclear transfer, which produced three cloned kids (K179/MSTN ${ }^{-1}$, K52-2/MSTN ${ }^{+/-}$, and $\mathrm{K} 52-1 / \mathrm{MSTN}^{+/+}$).

Conclusions: The results suggested that the MTAL-2 could disrupt MSTN efficiently in the goat genome. The mutated somatic cells could be used to produce MSTN-site mutated goats without developmental disruption. Thus, TALENs is an effective method for accurate genome editing to produce site-modified goats.
\end{abstract}

Keywords: Myostatin, TALENs, Mutational goat, Muscularity, Nuclear transfer

\section{Background}

Myostatin (encoded by the MSTN gene) is a member of the transforming growth factor family that acts as an important negative regulator of skeletal muscle growth $[1,2]$. Mutations in MSTN can inactivate its expression and result in a nonfunctional protein, which has great potential to enhance muscle growth, leading to dramatic muscularity and a "double-muscling" phenomenon in many species, including cattle [3], mice [4], humans [5], and sheep [6]. Recent studies showed that inhibiting MSTN increases skeletal muscle mass, reduces fat mass, and inhibits diet-induced and genetic obesity [7-9], providing an opportunity to improve the production of meat in livestock. Several research institutions have attempted to produce MSTN knockout goats or sheep [9-13]. However, because of the low rate of conventional homologous recombination or zinc-finger nuclease (ZFN)-

\footnotetext{
* Correspondence: chengyong@yzu.edu.cn

College of Veterinary Medicine, Yangzhou University, No. 12 Wenhui Road, Yangzhou 225009, Jiangsu Province, People's Republic of China
}

mediated knockout, there have been few reports of successful MSTN knockout goats [13].

Recently, the newly developed transcription activator-like effector nucleases (TALENs) and clustered regularly interspaced short palindromic repeats (CRISPR) techniques have emerged as powerful genetic tools to edit the genome precisely [14-17]. They represent a promising approach for targeted knockout at specific genomic loci, and have been used widely to perform precise genome editing in a wide range of organisms $[15,18-20]$. Recent advances in the study of the CRISPR system have resulted in improved efficiency that allowed it to rapidly replace other genome editing platforms, including ZFNs and TALENs. Outstanding work has been done by one team of researchers using CRISPR/Cas9 to edit the goat genome. In that work, they produced MSTN mutated founders. These founders should be valuable for research into MSTN. At same time, some off-target modifications were detected in their founders [11]. However, as new breeds of goat for human consumption, we must avoid or reduce the risk of off-target mutations as much as possible. Some reports showed that no or few off-target mutations were detected in the animals 
created by TALENs [14, 21, 22]. The latest report also showed that the cloned cattle created by TALENs combined with SCNT are free of off-target events [23]. Thus, to reduce or avoid the induction of off-target mutations, we chose the TALEN approach for MSTN editing in goats. As far as we know, MSTN-knockout in goats using TALENs has not been reported.

In this paper, we tested whether direct MSTN-editing mediated by TALEN in goat cells is a viable approach to produce MSTN mutant goats. To accomplish this, we considered two objectives: (i) to design functional MSTNmodified TALENs; and (ii) to develop methods for TALEN-mediated MSTN modification in a cloned goat.

\section{Results}

\section{Evaluation of TALENs efficiency}

We designed and assembled four pair of TALENs (Additional file 1: Table S1) each to target the first exon, first intron and third exon of goat MSTN. Each TALEN pair was transfected into primary GFFCs (goat fetus fibroblast cells), and PCR was used to measure the efficiency of genome modification was measured at $72 \mathrm{~h}$ after transfection. Sequencing and restriction analyses of the amplicons revealed that one pair of TALENs (MTAL-2, targeting the first exon) resulted in detectable gene-editing activity. A schematic and the binding sequences of MTAL- 2 are shown in Fig. 1a. Restriction analysis showed that the restriction enzyme could not completely cut the PCR amplicon from the mixed cells, when co-transfected with MTAL-2 expression plasmids (Fig. 1b). The sequencing ideograms of the cells transfected with MTAL-2 showed multiple peaks after the targeting site (Fig. 1c). The TAcloning sequencing showed 7 of 36 TA-cloning were detected with mutation. The frequency of mutated alleles was estimated at about $19.4 \%$.

\section{Generation of MSTN-modified goat fetal fibroblasts}

Eleven days after transfection with MATL-2, 320 monoclonal cell strains were randomly isolated. An AluI restriction site was designed in the MTAL-2 cut site. Genotyping was performed by PCR amplification, AluI digestion, and TA-cloning sequencing. Additional bands were observed by PCR amplification of the target region in some of the cell strains (Fig. 2a), indicating that genomic modification had occurred in these cells. As shown in Fig. 2a, when the PCR products were digested with AluI, two bands were observed in wild-type cell strains, and a 444-bp additional band was observed in mono-
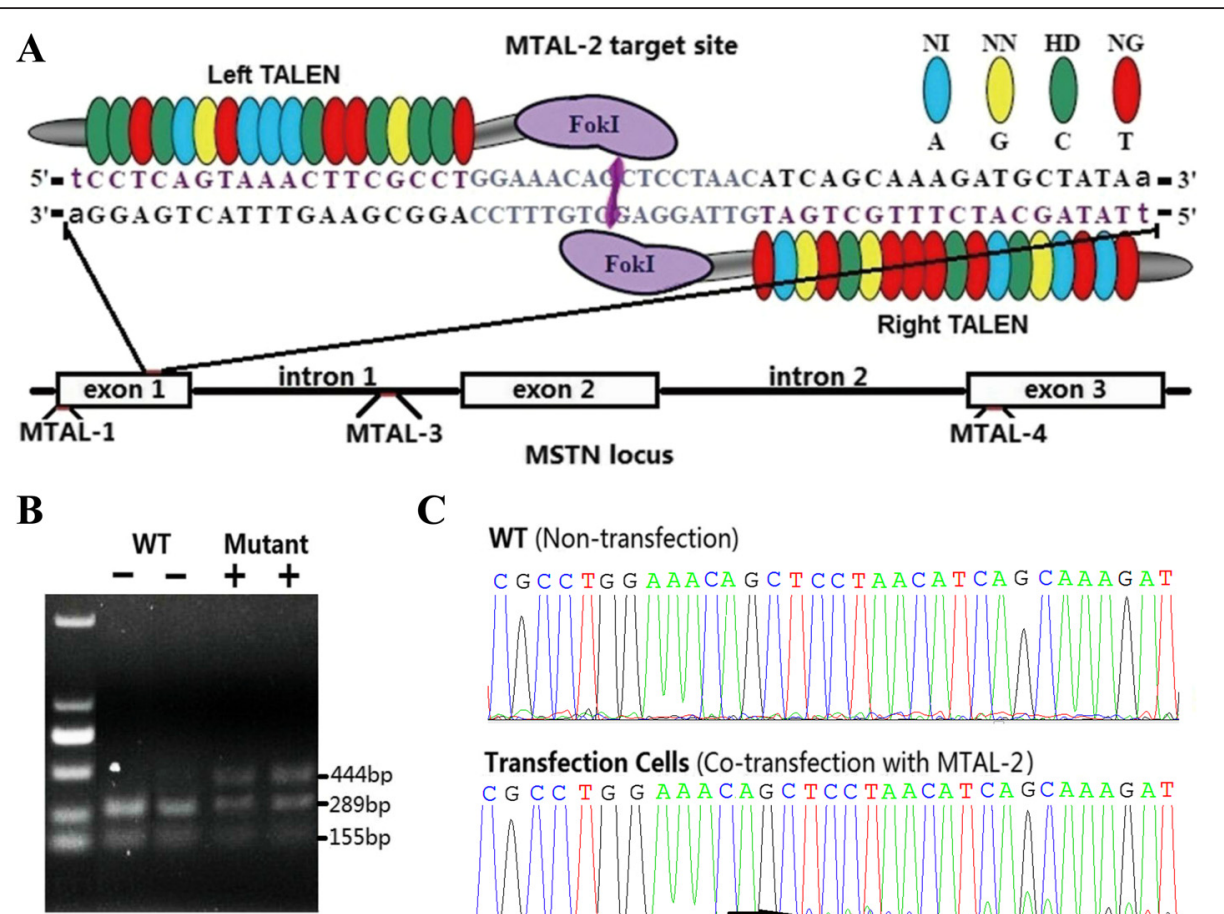

C

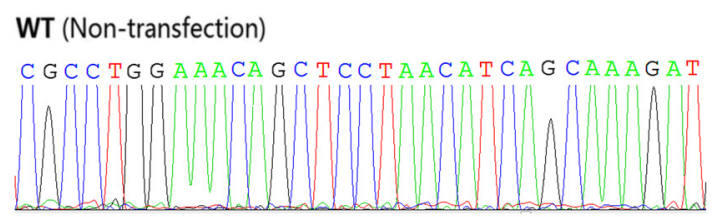

Transfection Cells (Co-transfection with MTAL-2)

C G C C T G G AAACAGC TCCTAACATCAGCAAAGAT

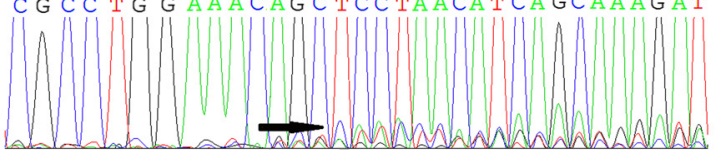

Fig. 1 Design of the TALENs assay targeting the goat MSTN locus. a Schematic showing the DNA-binding sites and spacer sequence of MTAL-2 in MSTN exon 1. b Modification of MSTN in GFFCs when transfected with one pair of functional TALENs (MTAL-2). Surveyor nuclease cleavage of the unsorted cells' PCR products produced an expected 444-bp band, while WT cells produced a 289-bp band and a 145-bp band. '- -' means no additional band was observed; ' ++ ' means the additional band was observed. c Mutation identified in mixed transfection cells. DNA fragments surrounding the TALEN target sites were analyzed by direct DNA sequencing. The sequencing ideogram shows a series of peaks after the targeting site 


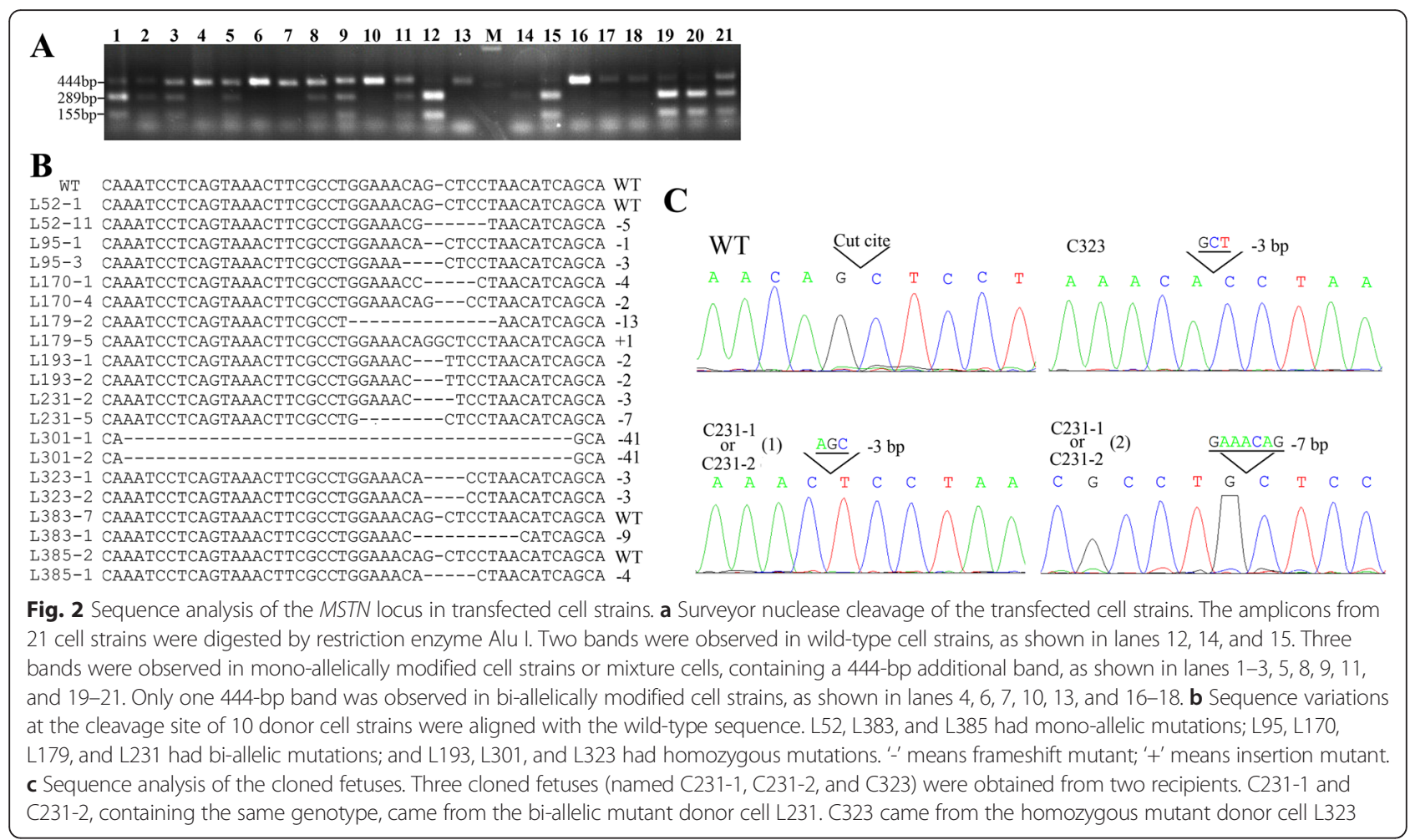

allelically modified cell strains, while only one 444-bp band was observed in bi-allelically modified cell strains. The results indicated that $272(85 \%)$ cell strains were modified at the MSTN locus. Among these cells strains, 174 (64 \%) were mono-allelically modified and 98 (36 \%) were bi-allelically modified (Table 1 ). TA-cloning sequencing of all bi-allelically modified cell strains and 34 wellgrown, mono-allelically modified cell strains showed that MTAL-2 induced small insertions or deletions in the target site, as shown in Fig. 2b. Three cell strains were identified as having homozygous mutations (Fig. 2b).

\section{Generation of MSTN mutant fetuses and goats}

To produce MSTN mutant goats, 10 well-grown cell strains with different sexes and genotypes were selected for use as donor cells for somatic cell nuclear transfer (SCNT). As shown in Fig. 2b, three cell strains were mono-allelically modified, four were bi-allelically modified,

Table 1 Genotype analyses of MSTN-modified goat fetal fibroblasts

\begin{tabular}{llll}
\hline $\begin{array}{l}\text { Genotype of } \\
\text { monoclonal cells }\end{array}$ & Count & Percentage (\%) & $\begin{array}{l}\text { Homozygous } \\
\text { mutations }\end{array}$ \\
\hline Total & 320 & & \\
Mono-allelic mutant & 174 & 54.4 & - \\
Bi-allelic mutant & 98 & 30.6 & $3(1 \%)$ \\
Wild type & 48 & 15 & - \\
\hline
\end{tabular}

and three were homozygously modified. Four hundred and three embryos were reconstructed and transferred into 29 synchronized recipients. Seven (24\%) recipients were confirmed as pregnant by transrectal ultrasonography 30 days after embryo transfer. Three fetuses were obtained from three pregnant recipients derived from donor cells of different genotypes (Fig. 2b). The fetal cell lines (named C231-1, C231-2 and C323) were isolated and expanded for use in recloning. Sequence analyses showed the all the fetuses had the same genotype as their donor cells. C231-1 and C231-2, derived from L231, had the same genotype and were modified biallelically. C323, derived from L323, was modified homozygously (Fig. 2b and c). The results suggested that TALEN-mediated MSTN-modified cells could support early embryonic development, and the mutations could be stably inherited.

Two pregnant recipients carried their kids to term, delivering three female kids (named K179, K52-1, and K52-2; weighing $5.8 \mathrm{~kg}, 3.4 \mathrm{~kg}$, and $4.5 \mathrm{~kg}$, respectively) (Fig. 3a-c). However, K179 died because of a difficult labor and produced edema, K52-1 died $1 \mathrm{~h}$ after cesarean section, and K52-2 is currently alive and healthy (Fig. 3f). Restriction enzyme digestion showed K179 (derived from L179) was bi-allelically modified, and K52-2 (derived from L52) was mono-allelically modified, while K52-1 had no mutation (Fig. 3g). The results suggested that MSTNmodified cells produced by TALENs could support embryonic development, resulting in MSTN-modified goats. 
A
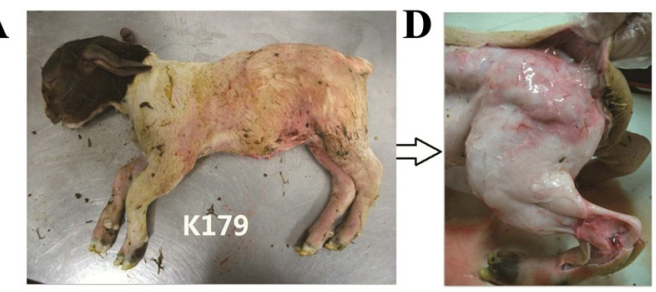

B

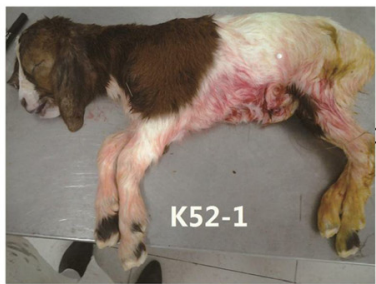

E

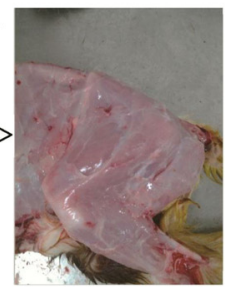

C
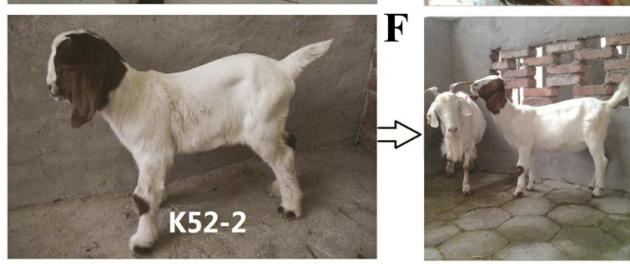

$\mathbf{L}$

AGT AAA CTT CGC CTG GAA ACA GCT CCT AAC ATC AGC AAA GAT wild-type sequence

K179 (1) AGT AAA CTT CGC CTG GAA ACA GGC TCC TAA CAT CAG CAA AGA O1 bp (substitution)+aa change

K179 \{ (2) AGT AAA CTT CGC --- --- -- --- -CT AAC ATC AGC AAA GAT $\triangle 13$ bp (frameshit)+ aa change

K52-1 $\{$ (1) AGT AAA CTT CGC CTG GAA ACA GCT CCT AAC ATC AGC AAA GAT WT

K52-1\{ (2) AGT AAA CTT CGC CTG GAA ACA GCT CCT AAC ATC AGC AAA GAT WT

K52-2\{(1) AGT AAA CTT CGC CTG GAA ACA GCT CCT AAC ATC AGC AAA GAT wT

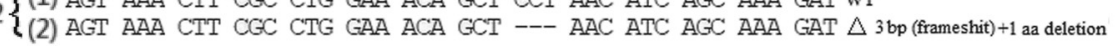

Fig. 3 Phenotype and genotype analysis of cloned goats. a-c Photographs of the cloned goats at the first day after birth. d, e Phenotypes of $\mathrm{K}_{179 / \mathrm{MSTN}^{-/-} \text {and K52-1/MSTN }}^{+/+}$. The leg portion of the carcass. Pictures of the femoris muscles of skinned cloned goats are shown. $\mathbf{f}$ Picture of the live goat K52-2 at 4 months. g Mutation confirmation in the cloned goats. $\mathbf{h}-\mathbf{k}$ Sequence analysis of the cloned goats. I Amino acid sequence analysis of the cloned goats

\section{Characterization of cloned goats}

Sequence analyses of the cloned kids showed that a 1-bp insertion and a 13-bp deletion were present in the target site of the K179 kid (Fig. 3i and j), and a 3-bp deletion was detected in one allele of the K52-2 kid (Fig. 3k). The mutations in K179 were predicted to result in a null mutation because they occurred after the first 68 and 65 amino acids of the C-terminal region, respectively, resulting in the loss of 307 and 310 amino acids (Fig. 31). The mutation in K52-2 resulted in a proline loss in the mature region of the protein (amino acid 70, Fig. 31). Western blot analysis further demonstrated that myostatin was not expressed in K179 and K52-2 showed lower myostatin expression than K52-1 (Fig. 4a).

MSTN mutations in goats might lead to increased muscularity. Compared with the wild-type cloned kid K52-1/ $\mathrm{MSTN}^{+/+}, \mathrm{K} 179 / \mathrm{MSTN}^{-/-}$showed a dramatic and widespread increase in skeletal muscle (Fig. $3 \mathrm{~d}$ and e). The body weight of $\mathrm{K} 179 / \mathrm{MSTN}^{-1-}$ was 1.7 times higher than $\mathrm{K} 52-1 / \mathrm{MSTN}^{+/+}$, although this result could not be accurately estimated because of edema. Compared with the wild-type littermate $\mathrm{K} 52-1 / M S T N^{+/+}$, the body weight of $\mathrm{K}^{2} 2-2 / \mathrm{MSTN}^{+/-}$was increased by $32 \%$ weight and the mean fiber area increased by $8.8 \%(P<0.05$, Fig. $4 \mathrm{~b}$ and c). Furthermore, K52-2/MSTN ${ }^{+-}$showed the same frequency distribution of myofibers (Fig. 4d) as was demonstrated in MSTN-knockout mice [24]. These results suggested that we generated a novel method to block MSTN expression in goats, which has potential to improve goat meat production.

\section{Off-target analysis}

To assay the specificity of MTAL-2 induced DNA disruption, we employed the protocol described by Yong $\mathrm{L}$ et al. [25] to scan the genomic sequences in goats. The spacers between the two effector-binding elements in all the potential off-target cleavage sites were greater than 100bp, which were too long to form Fok I dimerization. Thus, the PCR test that was performed in previous studies to confirm off-target sites was unnecessary [25-28]. In addition, the six most potential off-target cleavage sites were predicted (Additional file 2: Table S3) and analyzed 


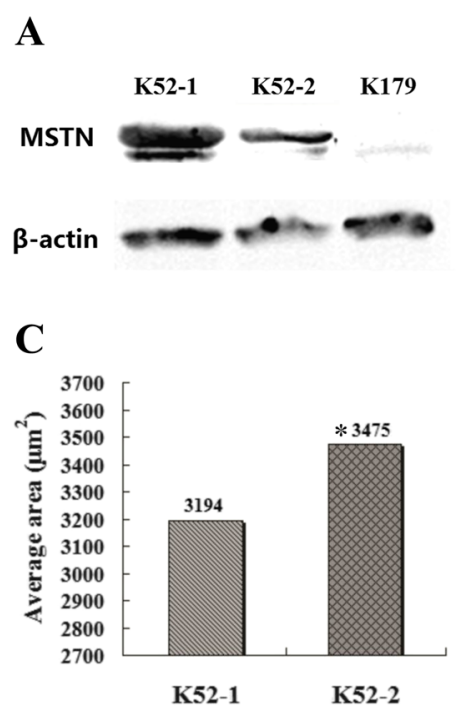

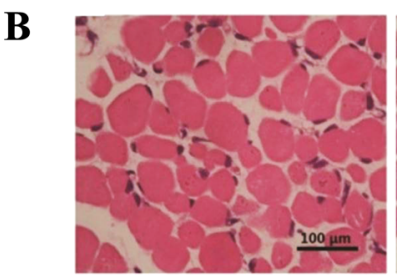

$\mathrm{K}^{2} 2-1 / \mathrm{MSTN}^{+/+}$

D

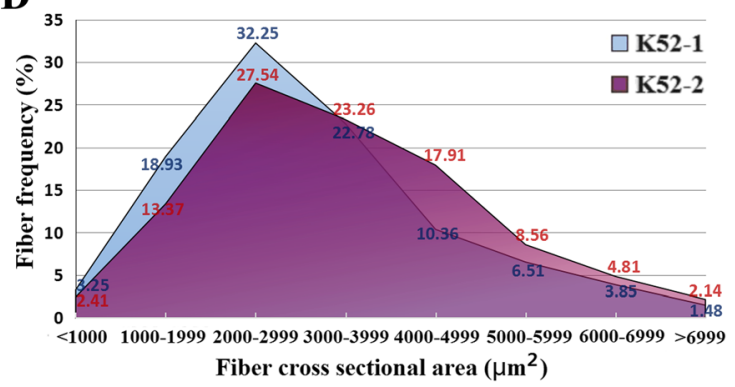

Fig. 4 Myofibers and western blot analysis of MSTN-targeted goats. a Myostatin protein expression of K179, K52-1, and K52-2 was normalized to $\beta$-actin expression using western blot assays. b Muscle cross-sections of $\mathrm{K} 52-2 / \mathrm{MSTN}^{+/-}$and $\mathrm{K} 52-1 / \mathrm{MSTN}^{+/+}$. Tissue sections were prepared from the fore limb tricep muscles that was dissected from the cloned goat $2 \mathrm{~h}$ after birth. $\mathbf{c}, \mathbf{d}$ Mean average area and frequency distribution of the myofibers in a given cross-sectional area. In the K52-2/MSTN ${ }^{+/-}$and K52-1/MSTN ${ }^{+/+}$groups, 338-374 randomly selected myofibers was measured and used for analysis. ${ }^{*} P$ value less than 0.05 considered as statistically significant. $\mathbf{d}$ The blue shaded area shows that more myofibers are in the 1000-3999 $\mathrm{\mu m}^{2}$ range in ${\mathrm{K} 52-1 / \mathrm{MSTN}^{+/+} \text {; the red shaded area shows that more myofibers are in the } 4000-6999 \mathrm{~mm}^{2} \text { range in K52-2/MSTN }}^{+/-}$

by DNA sequencing. No evidence of off-target cleavage events could be identified. The information on the offtarget loci and primer pairs used are listed in additional files (Additional file 2: Table S3 and Additional file 3: Table S4).

\section{Discussion}

In this study, one pair of functional TALENs was identified and was used to generate bi- or mono-allelic mutant cells, which were used as donor cells for SCNT, resulting in bi- and mono-allelic-modified goats. These results showed that the combination TALEN-mediated genome editing with SCNT is a useful method to accomplish MSTN mutagenesis in goats.

In recent years, developments in ZFNs, TALENs, and CRISPR technologies have emerged as alternatives to classical breeding and transgenic methods to improve livestock. When deciding which technology to use in an experiment, it is very important to consider the final application of the mutated animals [17]. In this research, we attempted to create new breeds of goat to improve muscle yield, with the ultimate goal of approval for human consumption. To avoid or prevent significant risks of offtarget mutations, we chose TALENs to achieve mutagenesis instead of CRISPR. Even though CRISPR has emerged recently as a potentially facile and efficient alternative to ZFNs and TALENs to induce targeted genetic alterations, CRISPR does not have better specificity than TALENs or ZFNs [17, 26]. Meanwhile previous studies have shown that low efficacy and minor off-target mutations may exist in TALEN-induced animals [22, 23, 28-31]. However, TALENs still provides a valuable option, thanks to its relatively high specificity [26]. Off-target analysis of the cloned goats revealed that no off-target sequences were detected. Additional studies will be required to evaluate the specificity of our functional TALENs.

Natural mutations in MSTN first exon [32], first intron [33], second exon [32], and third exon [34] have been found in animal populations, and all these mutations can lead to the double-muscle phenotype. Existing research shows that nucleotide variation among introns results in differential degrees of gene expression in eukaryotic cells [35]. In the present study, we tried to induce similar mutations in goat MSTN locus to knockout or reduce the expression of MSTN. The results indicated that MTAL-2, targeting for the first exon, could modify the MSTN locus. Most mutations induced by MTAL-2 were frameshifts in the target site, and the same mutation could be found at a high frequency in different cell strains. Cloned goats, derived from mutant cells with different sexes but containing the same mutation, can be used to produce homozygous mutant offspring by mating. In addition, the homozygous mutant cells can be used directly as donor cells for SCNT to produce homozygous animals.

SCNT is an important technology that has been used to clone certain livestock species [36]. Microinjection combined with TALEN mRNA is an efficient method that has been used to target MSTN in mice [37]. However, some reports stated that many of the resulting founder animals 
were chimeric, with multiple mutations [22, 27, 37]. The injection of TALENs into embryos is not as feasible in large animals as in small animals because large animals have longer gestation cycles and high recipient costs, which make it difficult to achieve large animal offspring with a single expected mutation [28]. The major advantage of SCNT over direct embryo injection with editor reagents is the predictable genotype of the offspring and the ability to generate clonal lines of edited animals [18]. Therefore, in this study, we chose expected genotype somatic cells modified by TALENs followed by SCNT to produce MSTN edited goats.

Three cloned goats derived from cells with the same genetic background were created. The MSTN-modified goats shared several hypermuscular features with MSTNmodified mice [37] or cattle. Our results showed that loss of myostatin did not disturb embryonic development, but led to increased body weight and mean fiber area.

\section{Conclusions}

TALENs construct MATL-2 provided efficient MSTN gene disruption in the goat genome. The MSTN-modified cells could be used as donor cells for SCNT to produce MSTN-site mutated goats without disruption of embryonic development. Loss of myostatin protein resulted in muscular hypertrophy. These results represent an effective way to produce "double muscle" goat breeds.

\section{Methods}

\section{Design and assembly of TALEN expression plasmids}

The first exon, third exon, and its immediate flanking regions of the Capra hircus MSTN gene (NG_EF588034.1) were scanned for putative TALEN-binding pairs using TAL Effector Nucleotide Targeter 2.0 [38]. Four pairs of TALEN-binding pairs were chosen to construct TALEN expression plasmids using the FastTALETM TALEN Assembly Kit (Shanghai, China; Cat. No. 1802-030). The TALEN expression backbone contains a CMV promoter for expression in mammalian cells and a puromycin selection cassette for transient enrichment of transfected cells expressing TALENs.

\section{Animals}

Yangtze River Delta white goats and Boer goats were kept in the Research Farm of Yangzhou University, China. All surgical procedures were performed in accordance with the Guiding Principles for the Care and Use of Laboratory Animals and were approved by the Institutional Animal Care and Use Committee of Yangzhou University.

\section{TALEN evaluation and edited cells}

Goat fetal fibroblasts isolation and culture methods were the same as those described in a previous study [39]. $2 \times$ $10^{6}$ cells were electroporated (Eppendorf multiporator) with $4 \mu \mathrm{g}$ of the right-arm-TALEN encoding plasmid and $4 \mu \mathrm{g}$ of the left-arm-TALEN encoding plasmid, under the following conditions: $400 \mathrm{~V}$ for $300 \mu$ s with one pulse. Empty backbone plasmid was used as a control. Puromycin $(3 \mu \mathrm{g} / \mathrm{mL})$ was added $24 \mathrm{~h}$ after transfection. Cells were collected at $72 \mathrm{hs}$ after transfection. The targeted regions were PCR amplified (Primer sequences were shown in Additional file 4: Table S2) from genomic DNA and the amplicons were sequenced.

To obtain mutant cell strains, the functional TALEN expression plasmids were co-transfected into male or female goat fetal fibroblasts. Puromycin-resistant cells were cultured for another 7 days in normal medium, after which monoclonal cells could be observed. A cloning cylinder (C1059, Sigma) was then loaded around the single cell colony, and the cells were digested with trypsin. The monoclonal cell strains were then subcultured on 48-well culture plates. A portion of the cell culture was collected for cryopreservation, and the remaining cells were used for PCR screening to confirm the presence of the mutation. The PCR products were analyzed by restriction analysis and sequencing, before and after cloning into TA vectors.

\section{Nuclear transfer}

The methods of animal treatment, oocyte collection, nuclear transfer, embryo transfer, and pregnancy status were as described in previous studies $[39,40]$. Ten cell strains of different sexes were used as donor cells for SCNT. Several cell lines containing a desired mutant genotype were isolated from pregnant recipients and expanded to be used as donor cells for the next round of SCNT.

\section{Characterization of cloned kids}

The cloned goats were weighed after birth, and their genomic DNA was extracted from the umbilical cord. To confirm the mutation, the PCR products of the cloned goats were analyzed by restriction analysis and sequencing before and after cloning into TA vectors. To observe the phenotype of the cloned goats, the dead goats were dissected and observed. The tricep muscles from the fore limbs in the cloned goats were isolated by a minimally invasive surgery at birth. Muscles were collected from dead goats and isolated from surviving goats by a minimal invasive procedure to make tissue sections and to extract proteins. The myofibers were subjected to straining and measured using the method reported in a previous study [24]. Muscle sections were photographed at $400 \times$ magnification. The muscle cross-sectional area was calculated using ImageJ software (National Institutes of Health, New York, USA). Data for myo-fibers were analyzed by a general linear model of regression using SPSS (General Linear Model, SPSS 11.0; SPSS, Inc., USA). Western blot analysis was conducted using a standard protocol. Antibodies for myostatin (Cat. No. ab98337), $\beta$-actin (Cat. No. ab8229), 
and goat polyclonal secondary antibody for rabbit IgG (Cat. No. ab97080) were purchased from the AbCam Company (AbCam, Inc., Cambridge, MA, USA).

\section{Off-target assay}

To verify whether off-target mutations occurred in cloned goats, the target sequence of the active TALENs was searched in web software (http://blast.ncbi.nlm.nih.gov/Blast.cgi). The potential off-target sites were evaluated by sequencing.

\section{Additional files}

Additional file 1: Table S1. TALEN target sequences. (DOC $35 \mathrm{~kb}$ )

Additional file 2: Table S3. Off-target sites assayed for MTAL-2.

(DOC $83 \mathrm{~kb}$ )

Additional file 3: Table S4. Primer pairs for PCR amplification of off-target regions for MTAL-2. (DOC $32 \mathrm{~kb}$ )

Additional file 4: Table S2. Primers for PCR amplification of TALENs target regions. (DOC $29 \mathrm{~kb}$ )

\section{Abbreviations}

GFFC, goat fetal fibroblast cell; MSTN, myostatin; SCNT, somatic cell nuclear transfer; TALEN, transcription activator-like effector nucleases

\section{Acknowledgments}

We thank Professor Lili Wu, Professor Huoying Shi, professor Xilong Xiong, and professor Qi Xu at the Yangzhou University in China, for their kind help during sampling clinical muscle and making tissue sections.

\section{Funding}

This work was supported by funds of the National Major Special Projects on New Cultivation for Transgenic Organisms (Grant No. 2011ZX08008-004 and 2009ZX08008-009B), Jiangsu Research and Innovation Project For Ordinary University Graduate (Grant No. CXZZ13-0915).

\section{Availability of data and materials}

The data supporting the results of this manuscript are included in the body of the manuscript and as supplemental data.

\section{Authors' contributions}

The project was designed by $Y C$ and BY. The design, assembly and identification of TALENs, isolation and identification of the mutant cell strains were performed by $B Y$ and TZ. The SCNT was carried out by $Y C, Y Y, B Y, S-Z S, R L, Z Q, B S, M Z$ and FM. Analyses of the cloned goats were carried out by BY. The article was written by BY and YC. All authors read and approved the final manuscript.

\section{Competing interests}

The authors declare that they have no competing interests.

\section{Consent for publication}

Not applicable.

\section{Ethics approval and consent to participate}

Animals were raised as per Institutional Animal Care and Use Committee of Yangzhou University. All surgical procedures were performed in accordance with the Guiding Principles for the Care and Use of Laboratory Animals and were approved by the Institutional Animal Care and Use Committee of Yangzhou University.

Received: 8 December 2015 Accepted: 15 July 2016

Published online: 27 July 2016

\section{References}

1. Lee SJ, McPherron AC. Regulation of myostatin activity and muscle growth. Proc Natl Acad Sci U S A. 2001;98(16):9306-11.
2. Yang X, Koltes JE, Park CA, Chen D, Reecy JM. Gene Co-Expression Network Analysis Provides Novel Insights into Myostatin Regulation at Three Different Mouse Developmental Timepoints. PLoS One. 2015;10(2):e0117607.

3. Kambadur R, Sharma M, Smith TPL, Bass JJ. Mutations in myostatin (GDF8) in Double-Muscled Belgian Blue and Piedmontese Cattle. Genome Res. 1997:7(1054-9803):6.

4. Nakatani M, Takehara $Y$, Sugino $H$, Matsumoto M, Hashimoto O, Hasegawa $Y$, Murakami T, Uezumi A, Takeda S, Noji S, et al. Transgenic expression of a myostatin inhibitor derived from follistatin increases skeletal muscle mass and ameliorates dystrophic pathology in mdx mice. FASEB J. 2008;22(2):477-87.

5. Stinckens A, Georges M, Buys N. Mutations in the myostatin gene leading to hypermuscularity in mammals: indications for a similar mechanism in fish? Anim Genet. 2011:42(3):229-34.

6. Guo T, Jou W, Chanturiya T, Portas J, Gavrilova O, McPherron AC. Myostatin inhibition in muscle, but not adipose tissue, decreases fat mass and improves insulin sensitivity. PLoS One. 2009;4(3):e4937.

7. Lin J, Arnold HB, Della-Fera MA, Azain MJ, Hartzell DL, Baile CA. Myostatin knockout in mice increases myogenesis and decreases adipogenesis. Biochem Biophys Res Commun. 2002;291(3):701-6.

8. Grobet L, Pirottin D, Farnir F, Poncelet D, Royo LJ, Brouwers B, Christians E, Desmecht D, Coignoul F, Kahn R, et al. Modulating skeletal muscle mass by postnatal, muscle-specific inactivation of the myostatin gene. Genesis. 2003;35(4):227-38.

9. Zhou ZR, Zhong BS, Jia RX, Wan YJ, Zhang YL, Fan YX, Wang LZ, You JH, Wang $Z Y$, Wang F. Production of myostatin-targeted goat by nuclear transfer from cultured adult somatic cells. Theriogenology. 2013;79(2):225-33.

10. Petersen B, Niemann H. Molecular scissors and their application in genetically modified farm animals. Transgenic Res. 2015;24(3):381-96.

11. Wang X, Yu H, Lei A, Zhou J, Zeng W, Zhu H, Dong Z, Niu Y, Shi B, Cai B, et al. Generation of gene-modified goats targeting MSTN and FGF5 via zygote injection of CRISPR/Cas9 system. Sci Rep. 2015;5:13878.

12. Liu C, Li W, Zhang X, Zhang N, He S, Huang J, Ge Y, Liu M. Knockdown of endogenous myostatin promotes sheep myoblast proliferation. In Vitro Cell Dev Biol Anim. 2014;50(2):94-102.

13. Zhang C, Wang L, Ren G, Li Z, Ren C, Zhang T, Xu K, Zhang Z. Targeted disruption of the sheep MSTN gene by engineered zinc-finger nucleases. Mol Biol Rep. 2014;41(1):209-15.

14. Hu J, Lei Y, Wong WK, Liu S, Lee KC, He X, You W, Zhou R, Guo JT, Chen X, et al. Direct activation of human and mouse Oct4 genes using engineered TALE and Cas9 transcription factors. Nucleic Acids Res. 2014;42(7):4375-90.

15. Niu J, Zhang B, Chen H. Applications of TALENs and CRISPR/Cas9 in Human Cells and Their Potentials for Gene Therapy. Mol Biotechnol. 2014;56(8):681-8.

16. Park KE, Telugu BP. Role of stem cells in large animal genetic engineering in the TALENs-CRISPR era. Reprod Fertil Dev. 2013;26(1):65-73.

17. Gaj T, Gersbach CA, Barbas lii CF. ZFN, TALEN, and CRISPR/Cas-based methods for genome engineering. Trends Biotechnol. 2013;31(7):397-405.

18. Whitelaw CBA, Sheets TP, Lillico SG, Telugu BP. Engineering large animal models of human disease. J Pathol. 2016;238(2):247-56.

19. Hsu PD, Lander ES, Zhang F. Development and Applications of CRISPR-Cas9 for Genome Engineering. Cell. 2014;157(6):1262-78.

20. Chen K, Gao C. Targeted genome modification technologies and their applications in crop improvements. Plant Cell Rep. 2013;33(4):575-83.

21. Sung YH, Baek IJ, Kim DH, Jeon J, Lee J, Lee K, Jeong D, Kim JS, Lee HW. Knockout mice created by TALEN-mediated gene targeting. Nat Biotechnol. 2013;31(1):23-4

22. Tesson L, Usal C, Menoret S, Leung E, Niles BJ, Remy S, Santiago Y, Vincent Al, Meng $X$, Zhang $L$, et al. Knockout rats generated by embryo microinjection of TALENs. Nat Biotech. 2011;29(8):695-6.

23. Carlson DF, Lancto CA, Zang B, Kim E-S, Walton M, Oldeschulte D, Seabury C, Sonstegard TS, Fahrenkrug SC. Production of hornless dairy cattle from genome-edited cell lines. Nat Biotech. 2016;34(5):479-81.

24. Wang K, Li Z, Li Y, Zeng J, He C, Yang J, Liu D, Wu Z. Muscle-specific transgenic expression of porcine myostatin propeptide enhances muscle growth in mice. Transgenic Res. 2013;22(5):1011-9.

25. Lei Y, Guo X, Liu Y, Cao Y, Deng Y, Chen X, Cheng CHK, Dawid IB, Chen Y, Zhao $H$. Efficient targeted gene disruption in Xenopus embryos using engineered transcription activator-like effector nucleases (TALENs). Proc Natl Acad Sci U S A. 2012;109(43):17484-9.

26. Teimourian S, Abdollahzadeh R. Technology developments in biological tools for targeted genome surgery. Biotechnol Lett. 2014;37(1):29-39. 
27. Song J, Zhong J, Guo X, Chen Y, Zou Q, Huang J, Li X, Zhang Q, Jiang Z, Tang C, et al. Generation of RAG 1- and 2-deficient rabbits by embryo microinjection of TALENs. Cell Res. 2013;23(8):1059-62.

28. Xin J, Yang H, Fan N, Zhao B, Ouyang Z, Liu Z, Zhao Y, Li X, Song J, Yang Y, et al. Highly Efficient Generation of GGTA1 Biallelic Knockout Inbred Mini-Pigs with TALENs. PLoS One. 2013;8(12):e84250.

29. Boettcher M, McManus Michael T. Choosing the Right Tool for the Job: RNAi, TALEN, or CRISPR. Mol Cell. 2015;58(4):575-85.

30. Wei C, Liu J, Yu Z, Zhang B, Gao G, Jiao R. TALEN or Cas9 - Rapid, Efficient and Specific Choices for Genome Modifications. J Genet Genomics. 2013;40(6):281-9.

31. Xu L, Zhao P, Mariano A, Han R. Targeted Myostatin Gene Editing in Multiple Mammalian Species Directed by a Single Pair of TALE Nucleases. Mol Ther Nucleic Acids. 2013;2:e112

32. Grobet L, Poncelet D, Royo LJ, Brouwers B, Pirottin D, Michaux C, Ménissier F, Zanotti M, Dunner S, Georges M. Molecular definition of an allelic series of mutations disrupting the myostatin function and causing doublemuscling in cattle. Mamm Genome. 1998;9(3):210-3.

33. Schuelke M, Wagner KR, Stolz LE, Hübner C, Riebel T, Kömen W, Braun T, Tobin JF, Lee S-J. Myostatin mutation associated with gross muscle hypertrophy in a child. N Engl J Med. 2004;350(26):2682-8.

34. Grobet L, Royo Martin LJ, Poncelet D, Pirottin D, Brouwers B, Riquet J, Schoeberlein A, Dunner S, Menissier F, Massabanda J, et al. A deletion in the bovine myostatin gene causes the double-muscled phenotype in cattle. Nat Genet. 1997;17(1):71-4.

35. He Y, Wu Y, Lan Z, Liu Y, Zhang Y. Molecular analysis of the first intron in the bovine myostatin gene. Mol Biol Rep. 2010;38(7):4643-9.

36. Srirattana K, Sripunya N, Sangmalee A, Imsoonthornruksa S, Liang Y, Ketudat-Cairns M, Parnpai R. Developmental potential of vitrified goat oocytes following somatic cell nuclear transfer and parthenogenetic activation. Small Rumin Res. 2013;112(1-3):141-6.

37. Zhou F, Sun R, Chen H, Fei J, Lu D. Myostatin gene mutated mice induced with tale nucleases. Anim Biotechnol. 2015;26(3):169-79.

38. Doyle EL, Booher NJ, Standage DS, Voytas DF, Brendel VP, VanDyk JK Bogdanove AJ. TAL Effector-Nucleotide Targeter (TALE-NT) 2.0: tools for TAL effector design and target prediction. Nucleic Acids Res. 2012;40(W1):W117-22.

39. An LY, Yuan YG, Yu BL, Yang TJ, Cheng Y. Generation of human lactoferrin transgenic cloned goats using donor cells with dual markers and a modified selection procedure. Theriogenology. 2012;78(6):1303-11.

40. Yuan YG, Cheng Y, Guo L, Ding GL, Bai YJ, Miao MX, An LY, Zhao JH, Cao YJ. Cloned kids derived from caprine mammary gland epithelial cells. Theriogenology. 2009;72(4):500-5.

\section{Submit your next manuscript to BioMed Central and we will help you at every step:}

- We accept pre-submission inquiries

- Our selector tool helps you to find the most relevant journal

- We provide round the clock customer support

- Convenient online submission

- Thorough peer review

- Inclusion in PubMed and all major indexing services

- Maximum visibility for your research

Submit your manuscript at www biomedcentral.com/submit

) Biomed Central 